\title{
DEVELOPMENT OF HISTORICAL AND CULTURAL THAMAR CITY CENTER IN REPUBLIC OF YEMEN
}

\author{
Associate Prof. Najib Ali Abdullah Al-Maqtari \\ Architectural Dep. Thamar University \\ Head of Projects Unit - Thamar University \\ E-mail: alnajeebcos@yahoo.com \\ Tele/fax : $+967-1-440813$ \\ E-mail : najeebmaqtari@hotmail.com \\ mobile : $+967-777120355$ \\ Republic of Yemen p.o Box : 13404 Sana'a
}

(Received August 17, 2011 Accepted November 3, 2011)

old historical cities are distinguished by beautiful architectural environment, fantastic natural landscapes and human architectural heritage which was existed due to long historical accumulations and this heritage is mixed with natural environment and they both constitute unique joint entity which resulted from cultural and social and economic civilizations for that cities.

Historical cities are violated in terms of beautiful features changes by horizontal and vertical additions for that arenas, spaces, markets and buildings and thamar city is like the other Yemen cultural and historical cities.

Through this research, we focus on highlighting the importance of historical cities and Thamar is example for the purpose of re-planning of main areas which will be the cornerstone for development and economic investment and attractive areas that activate the other parts of the city.

This research deals with three main topics, the first one is to present the problem and the second one is to re-plan the axis that links between the Great Mosque and Sun School, which passes through the center of the city and to remove additions and deformations and the last one is to propose establishing investment center, which is supposed to be like basis for preserving and developing the historical city.

The research reached to proposal that is aimed to open axis that links between great mosque and Sun School and to establish tourist and economic investment center to activate and rejuvenate historical cities.

KYWORDS: preservation, development, revival, investment, re-planning, historical city.

\section{تطوير مركز مدينة ذمار التاريخية والثقافية في الجمهورية اليمنية}

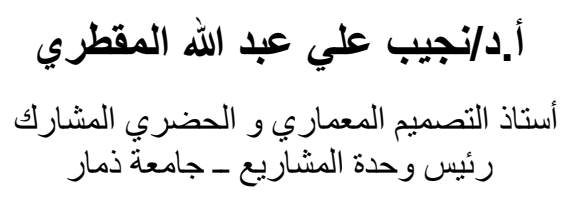

E-mail:alnajeebcos@yahoo.com

Tel/fax:+967-1-440813

E-mail:najeebmaqtari@hotmail.com Mobile:+967-777120355

Mobile:+967-711195510

Republic of Yemen P.O.BOX: 13404 sana'a 


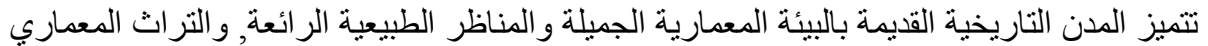

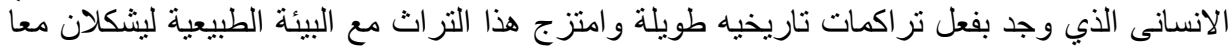

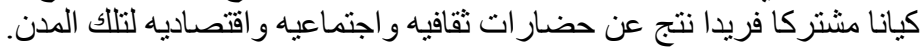

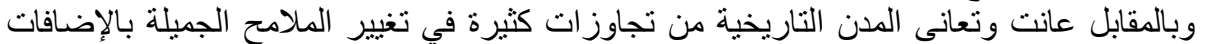

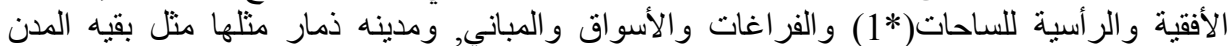
التاريخية الثقافية اليمنية.

ونهدف من خلال هذا البحث إلى تسليط الضوء الضئه على أهمية المدن التاريخية ومدينة ذمار كحالة

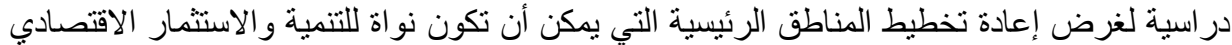
و مناطق جذب لتحريك الأجز اء الأخرى للمدينة.

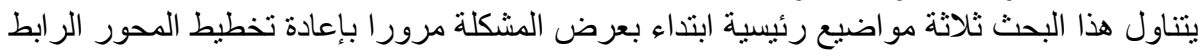

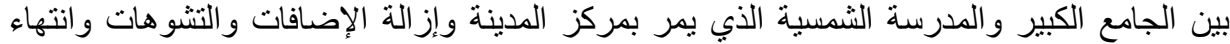
بمقترح إنشاء مركز استثماري يكون بمثابة إثتعاع للحفاظ وتطوير المئرية المدينة التاريخية.

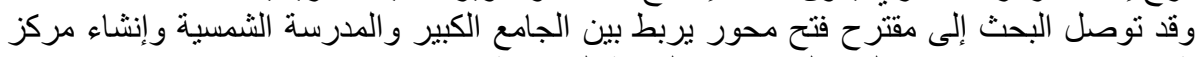

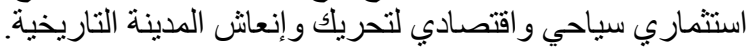
الكلمات ألمفتاحيه: الحفاظ , التطوير , الإنعاش , الاستثمار , إعادة التخطيط , المدينة التاريخية. .

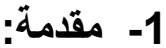

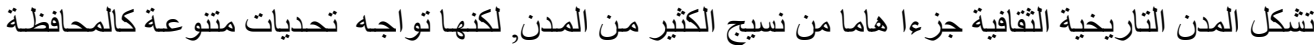

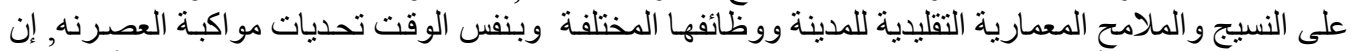

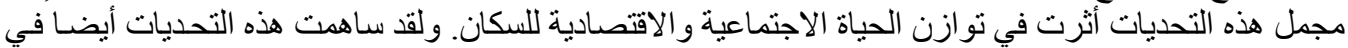

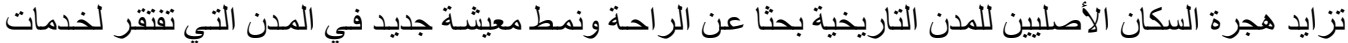

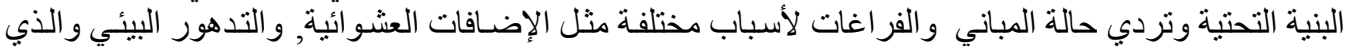

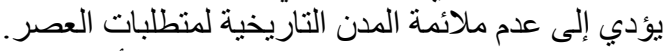

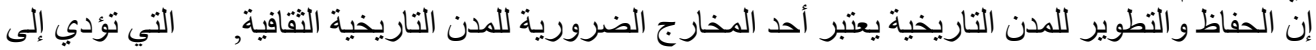

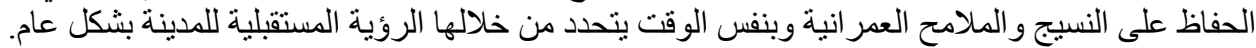

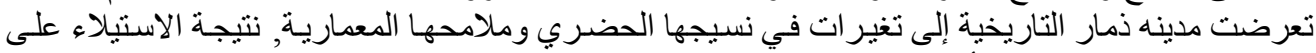

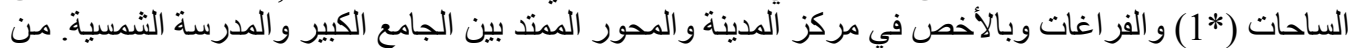

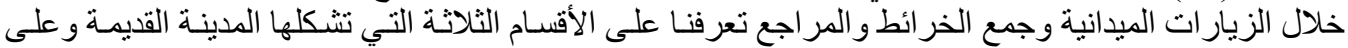

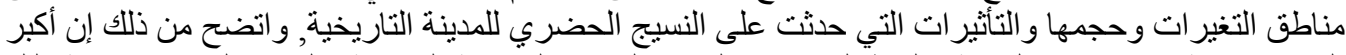

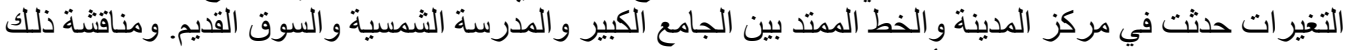

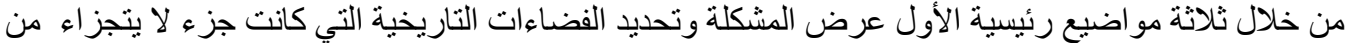

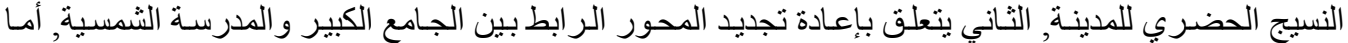

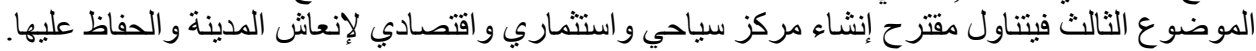

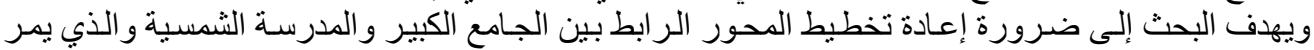

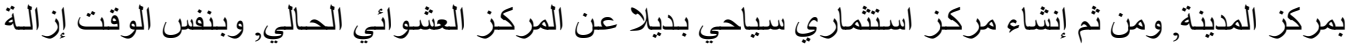
التشو هات من هذه المناطق.

\section{2- مشكلة الدراسة}

مدينة ذمار التاريخية تتكون من ثناثة إحياء حسب نشوئها التاريخي وتسمى:

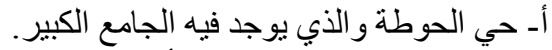

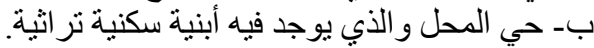

ج- حي الجراجيش الذي يوجد فيه المدرسة الثمسية وجامع داديه والسوق الفيه القديم, لكنة أكثر الأحياء

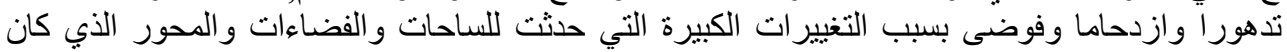


يربط بين الجامع الكبير و المدرسة الثمسية وكذلك التغيرات التي حدثت في السوق القديم و الساحات المحيطة

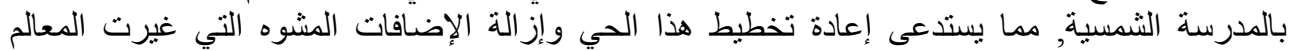
الحضرية للحي.

\section{3- هدف البحث}

يهدف البحث إلى در اسة العلاقة بين الثلاث المناطق التاريخية وهي بمدينة ذمار (وهي حي الحوطة وحي المحل

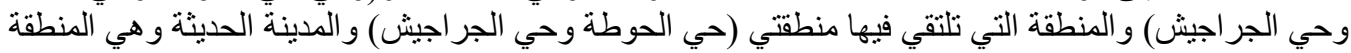

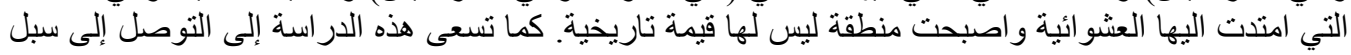
تتمية المنطقة وتتشيطها سياحيا وصياغتها بمظهر لائق.

\section{4 - منهجية البحث}

تعتمد الدراسة على المنهج التحليلي للنسيج الحضري والتشكيل العمراني للأحياء الثلاثة وجمع البيانات

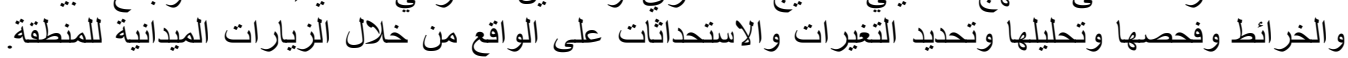

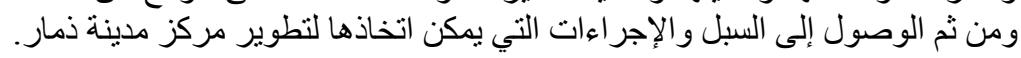

\section{5- عرض وتحليل منطقه الدراسة :}

\section{1-5 مكونات المدينة ومراحل تطور ها:}

تطورت مدينة ذمار خلال ثلاث مر احل تاريخية الأولى قبل العام 1962م و المرحلة الثانية كانت مابين العام

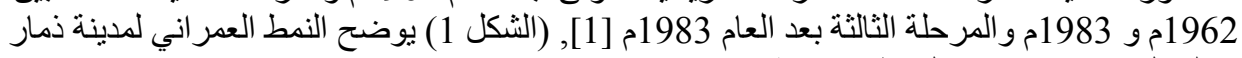

و(الشكل 2) يوضح مر احل تطور مدينة ذمار.

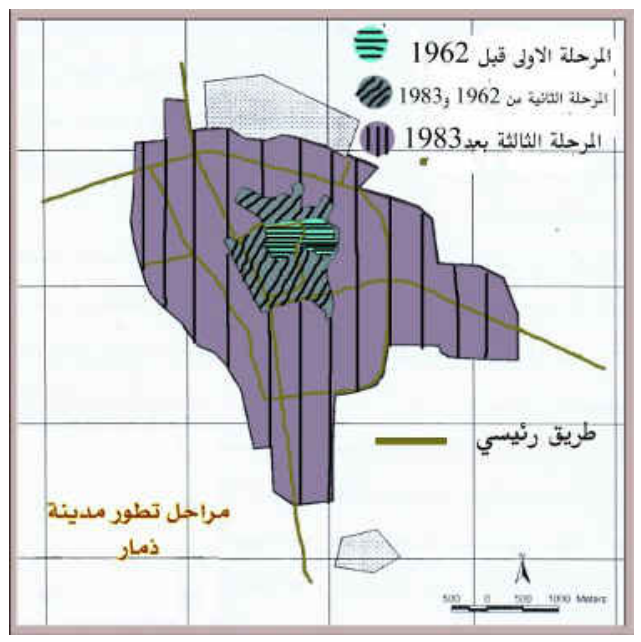

شكل (2) : مر احل تطور مدينة ذمار

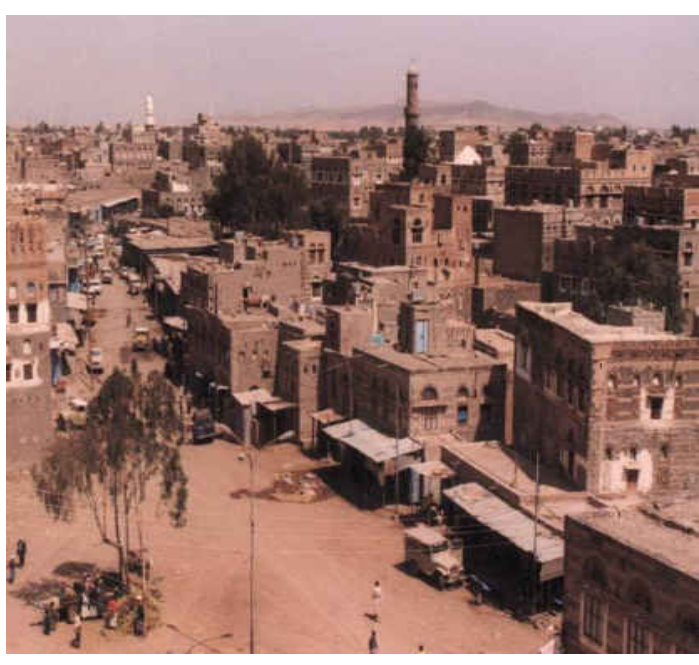

شكل (1) : صورة توضح النمط العمر اني لمدينة ذمار القديمة و تتكون مدينة ذمار القديمة من ثلاثة أحياء [2] هي:

$$
\text { • • • • • • }
$$

(الثكل3) يوضح موقع المدينة القيمة و(الثكل4) يوضح الأحياء الثناثة][]. 


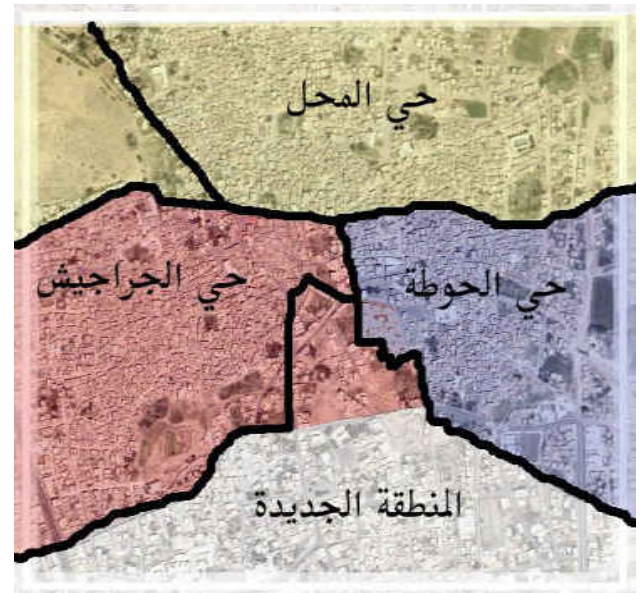

شكل (4) : الأحياء الثلاثة المكونة للمدينة القديمة

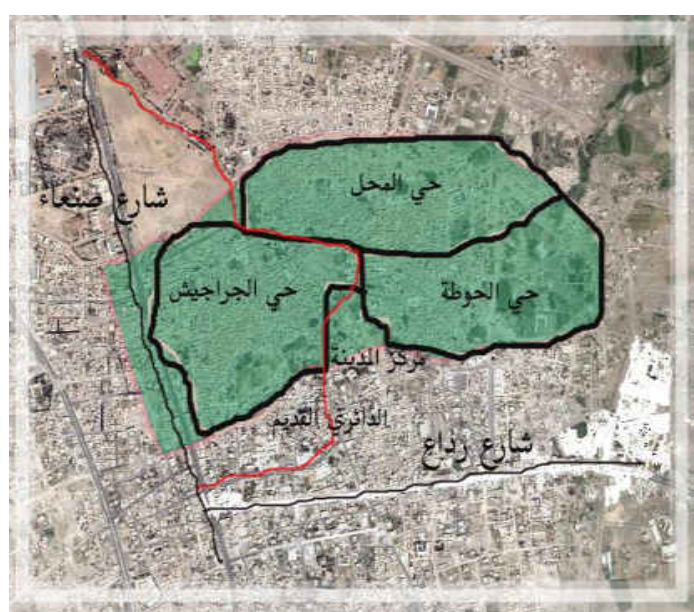

شكل (3): موقع المدينة القديمة ضمن مدينة ذمار

\section{-1-5 - 1- 1حي الحوطة}

يعتبر حي الحوطه من أهم وأقدم أحباء مدينة ذمار التاريخية، والذي يحتوي على عدد من الأبنية السكنية

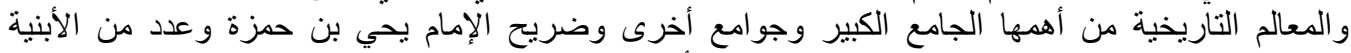

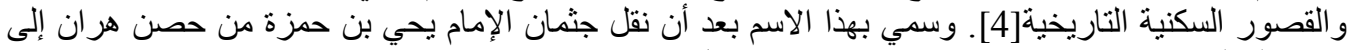
مكانه الحالي فسمي بحوطة الإمام يحي بن حمزة ( شكل 5 ) .
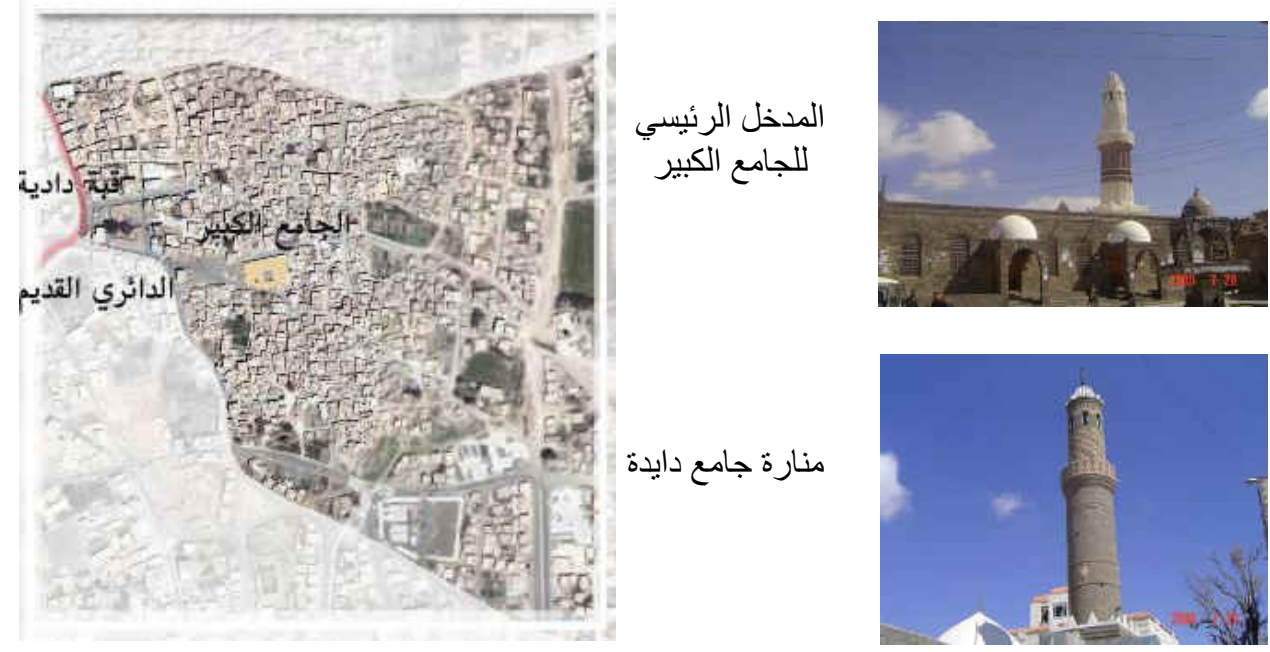

شكل (5) : خريطة توضح حدود حي الحوطة وموقع الجامع الكبير و الددخل الرئيس و المنارة ومنارة جامع دادية

$$
\text { 2-1-5 }
$$

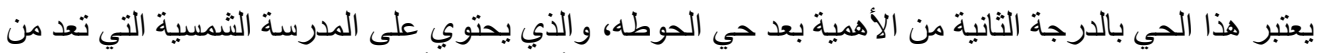

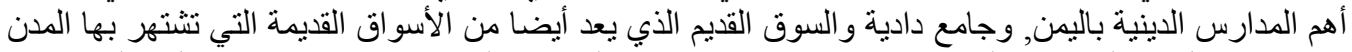

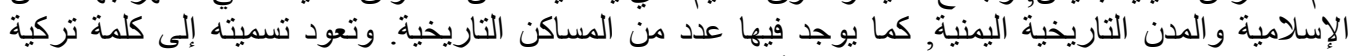

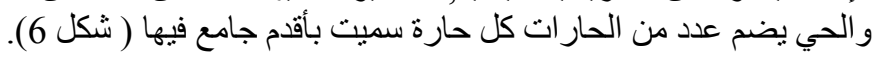




\section{3-1-5 حي المحل}

سمي هذا الحي بهذا الاسم نسبة إلى محل إقامة الو الي العثماني على اليمن أثر دخولهم الأول نهاية القرن العاثر

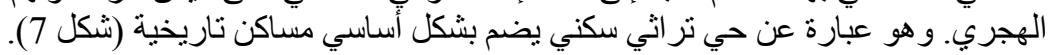

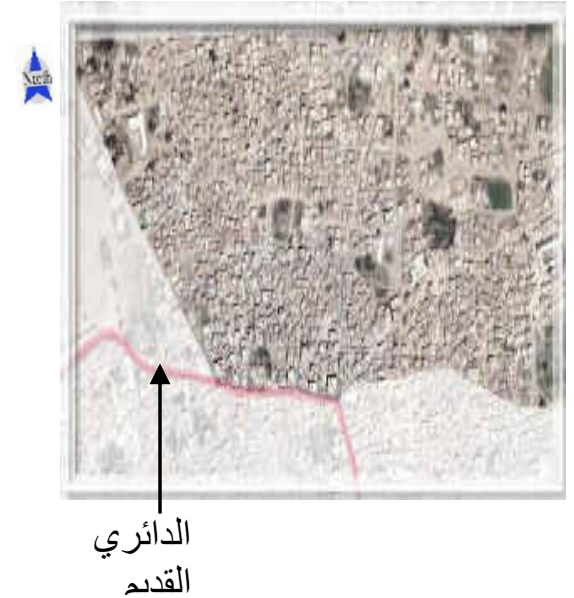

شكل (7) : خريطة توضح حدود حي الدحل

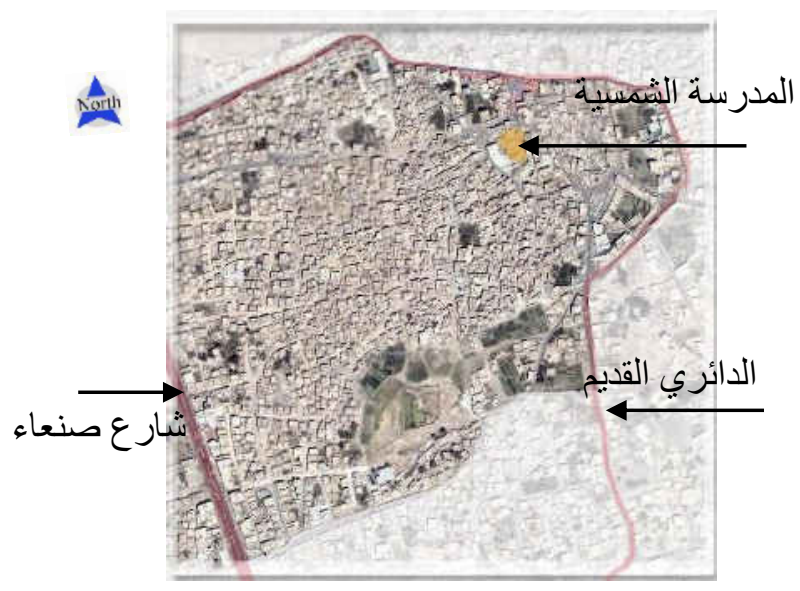

شكل (6) : خريطة توضح حدود وأهم معالم حي

4-1-5 (منطقة الاراسة) المنطقة التي تقع بين منطقتي حي الحوطة وحي الجراجيش ( مركز المدينة )

من خلال الدراسة الميدانية اتضح أن هذه المنطقة تكثر فيها المقاشم (2) التي تلبي الحاجات اليومية للسكان

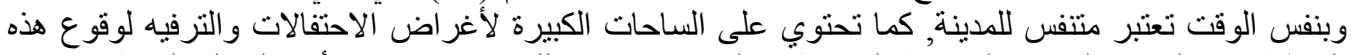

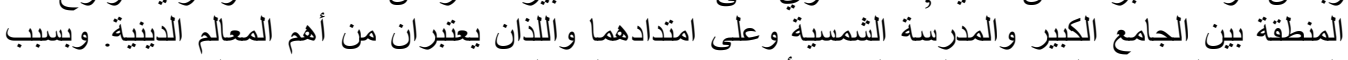

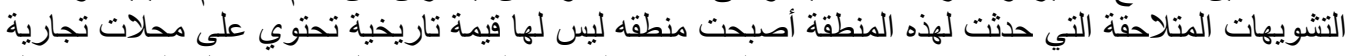

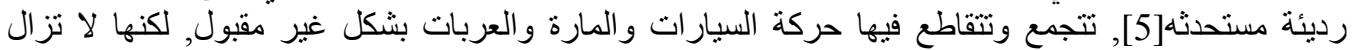

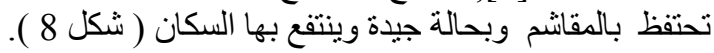
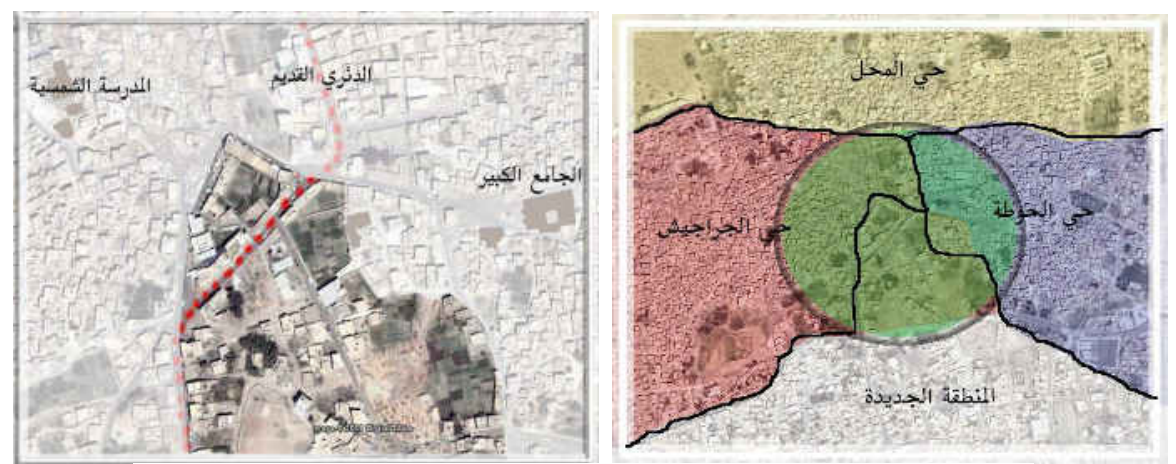

$$
\text { شكل (8) : خريطة توضح المنطقة الوسطية منطقة الدراسة }
$$




\section{2-5 مركز المدينة والمحور الرابط بين الجامع الكبير والمدرسة الثمسية}

بالتأكيد توجد علاقة بين الجامع الكبير والمدرسة الشمسية[6], لكن بسبب الاختلال الذي حدث في الساحات

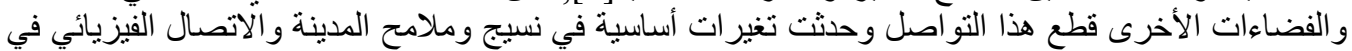

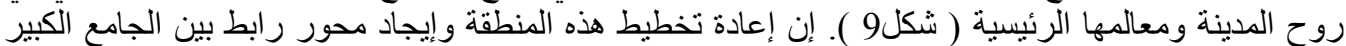

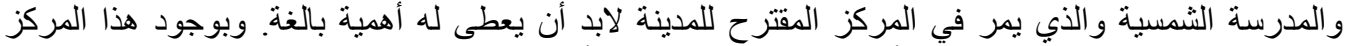

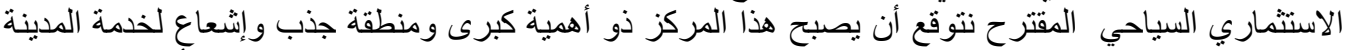

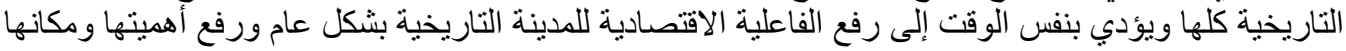

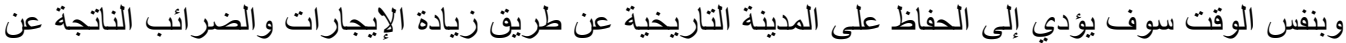
التوسع في الأبنية و المحلات و الترفية التئ.
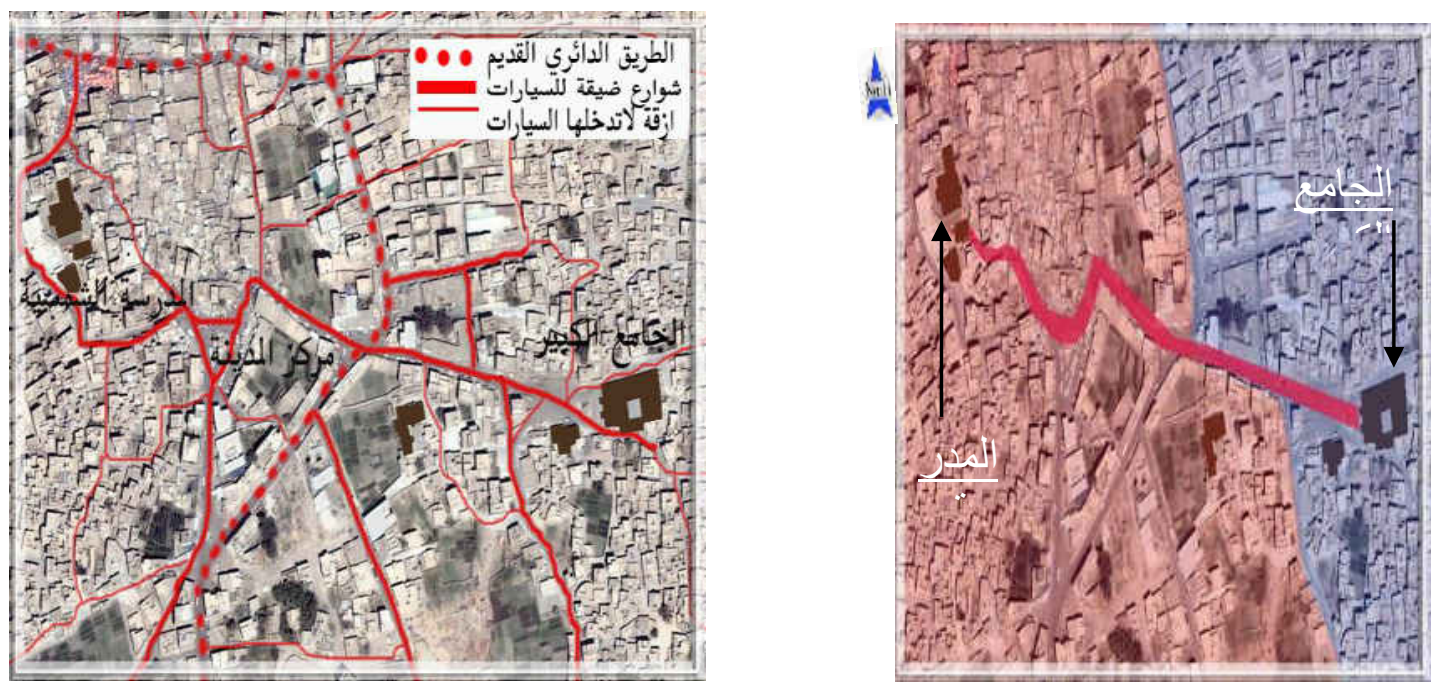

شكل (9) خريطة توضح المحور الر ابط بين الجامع الكبير و المدرسة الثمسية وشبكة الطرق

\section{الجامع الكبير والمدرسة الشمسية}

تعد مدينة ذمار واحدة من أهم المدن التاريخية اليمنية والتي تزخر بالأحياء التراثية السكنية و الأبنية التاريخية

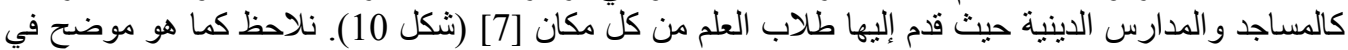

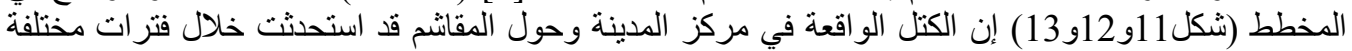

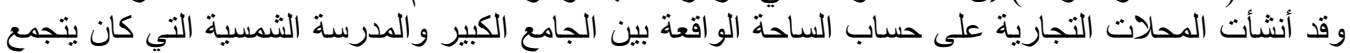

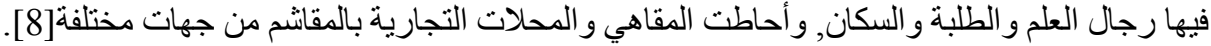

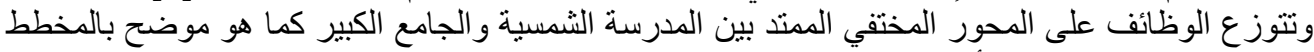

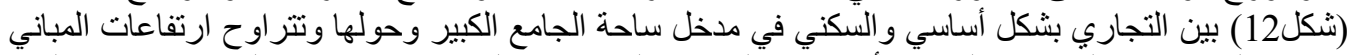

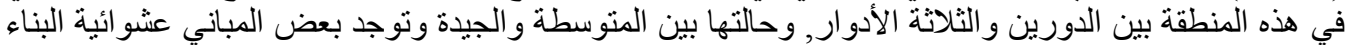

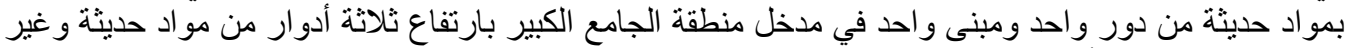

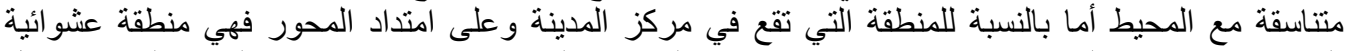

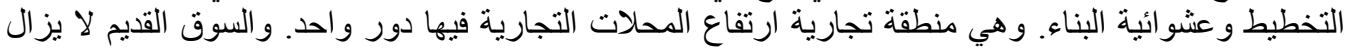

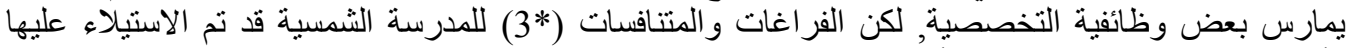

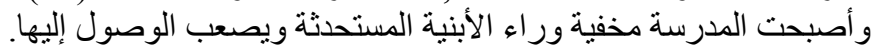




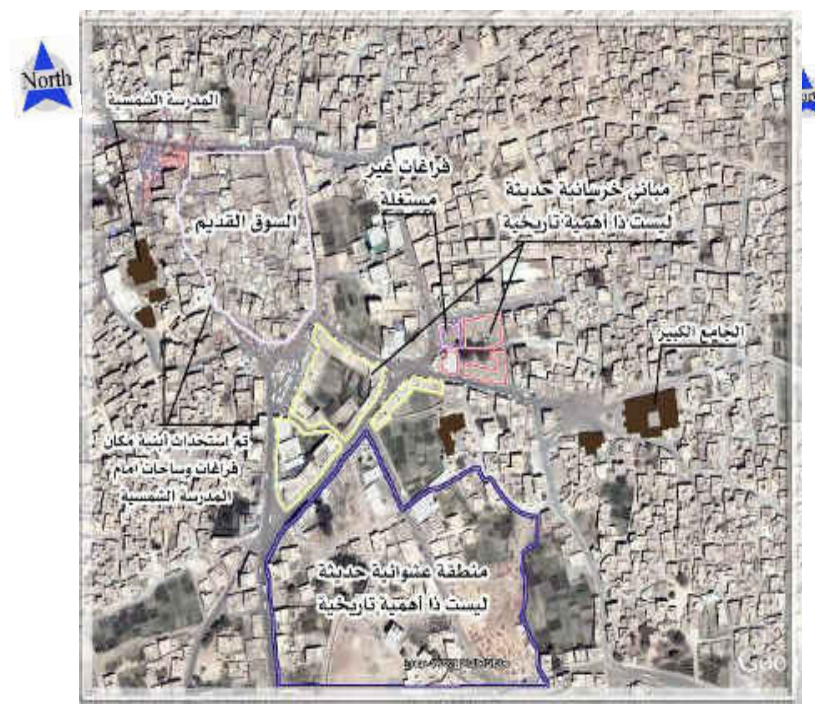

شكل (11) خريطة توضح حالة مركز الددينة

و المنطقة الممندة من الجامع الكبير حتى المدرسة

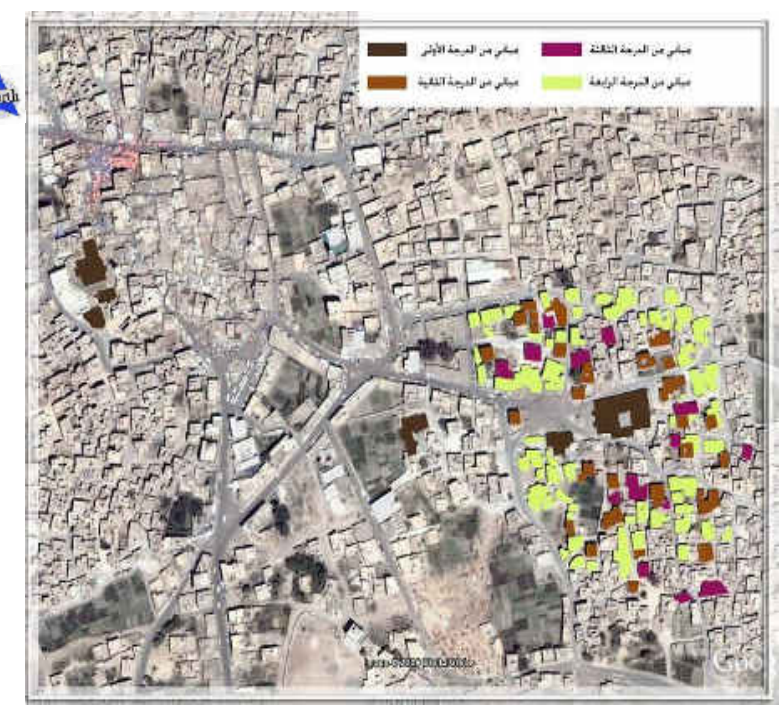

شكل (10) خريطة توضح الأهمية التاريخية

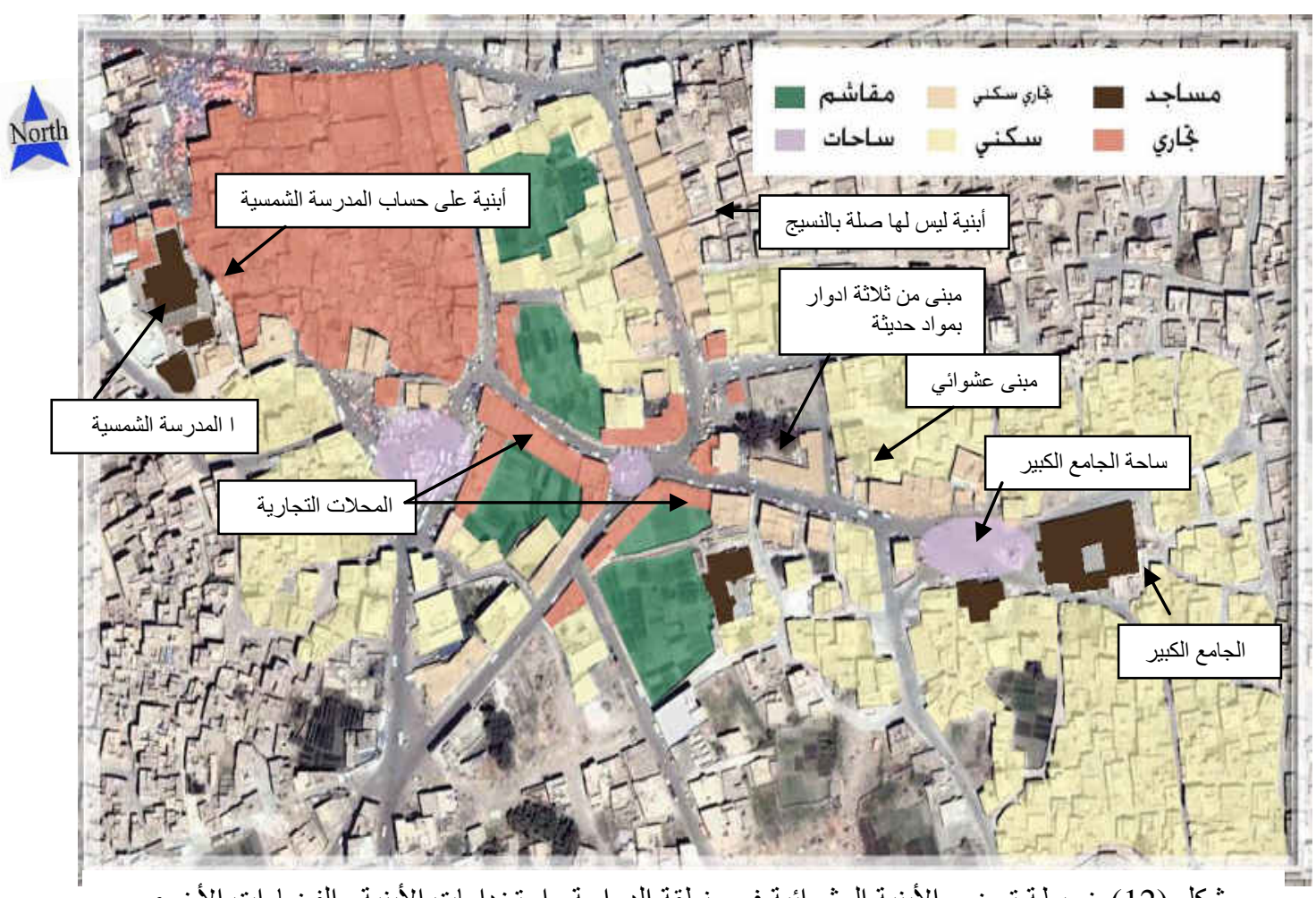

شكل (12) خريطة توضح الأبنية العشو ائية فى منطقة الدراسة واستخدامات الأبنية و الفضاءات الأخرى 
1-7 حالة منطقة الدراسة والروئة المستقبلية لها

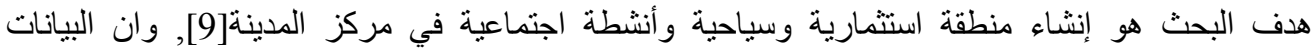

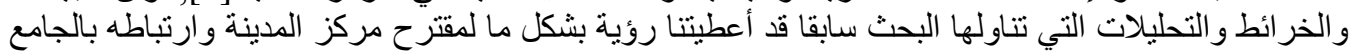

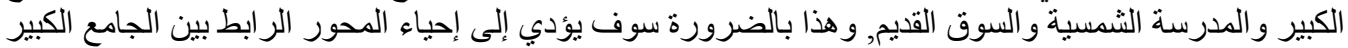
و المدرسة الثمسية و السوق الفئ القديم.

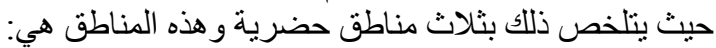

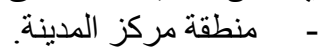
- - - منطقة الجامع الكبير وساحته الأمامية. - منطقة المدرسة الشمسية و السوق القديم.

وفيما يلي نرى إتباع المعالجات الآتية إز اء كل منطقة من المناطق الثلاث [10] ( شكل 13):

أـ المنطقة الوسطية مركز المبلة المدينة.

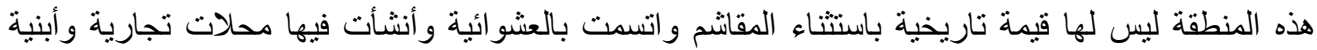

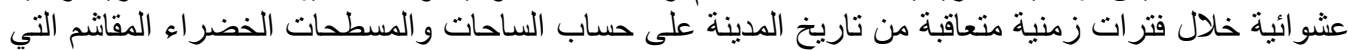

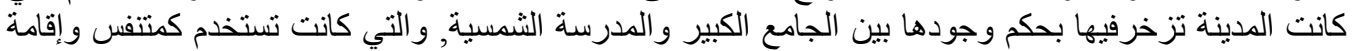

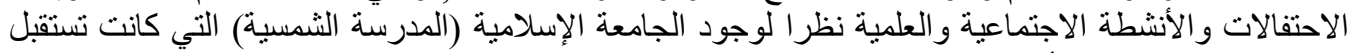

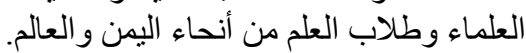

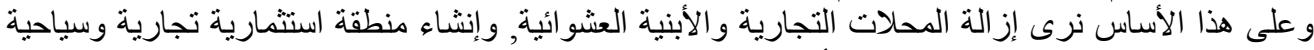

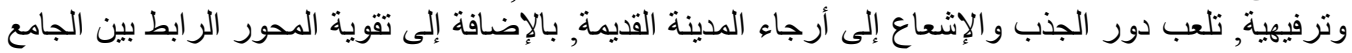

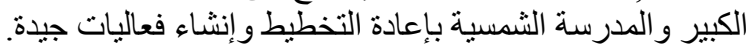

ب- منطقة الجامع الكبير وساحته الأمامية.

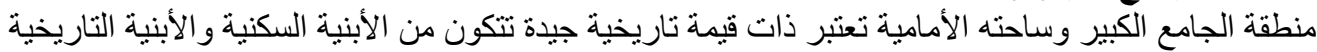

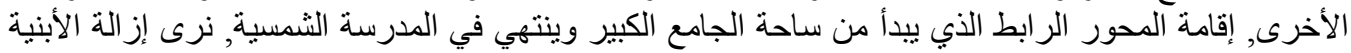

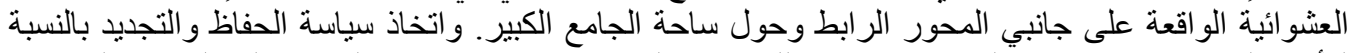

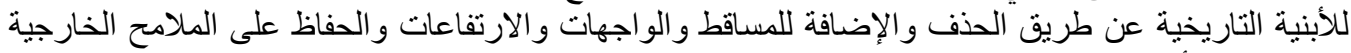
للو اجهات و الأسطح الخارجية و إتباع سياسة التحديث الداخلي للوظائف و الثبكات المختلفة.

ج- منطقة المدرسة الشمسية والسوق القديم.

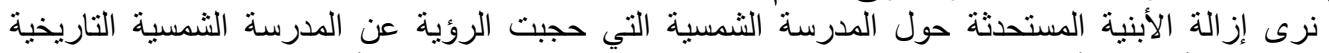

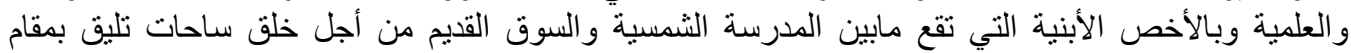

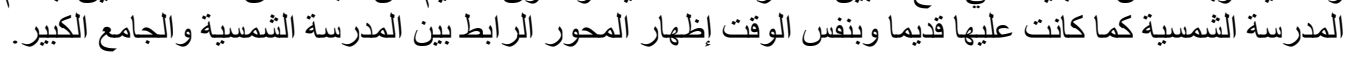




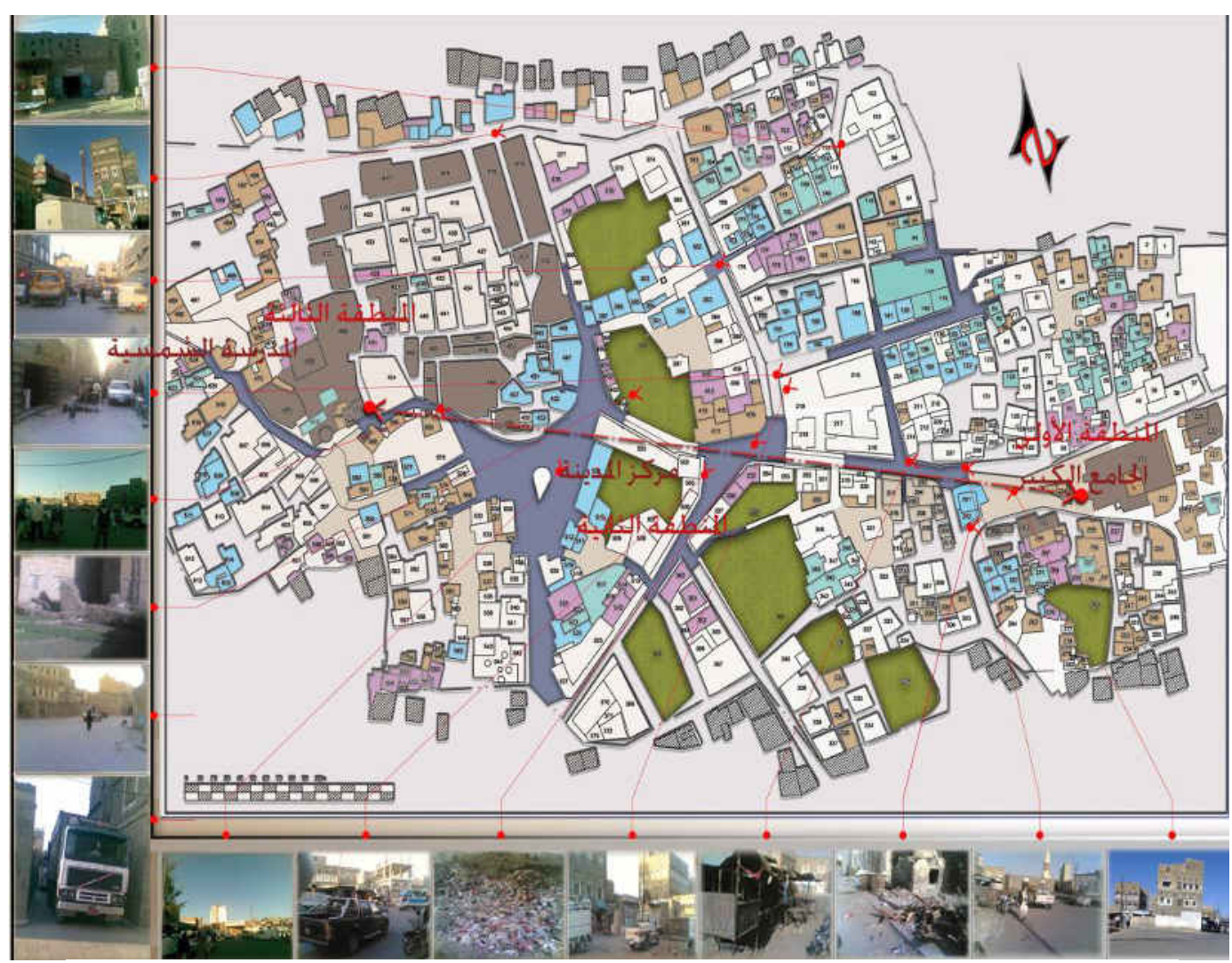

شكل (13) خريطة توضح الحالة الر اهنة لمنطقة الدر اسة و المناطق الثناث التي تشملها الدر اسة

2-7 مقترح تخطيط مركز المدينة وتجديد المحور الممتد بين الجامع الكبير والمدرسة الثمسية

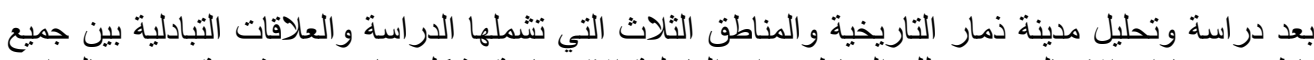

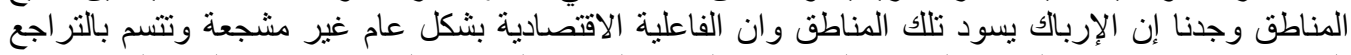

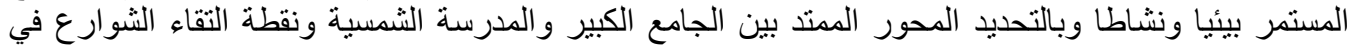

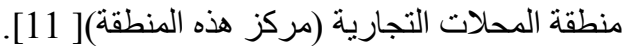

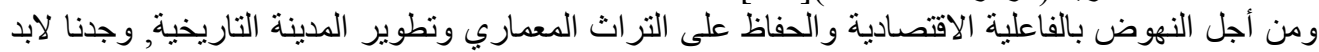

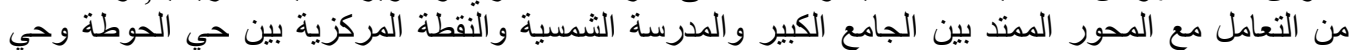

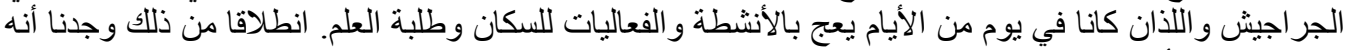

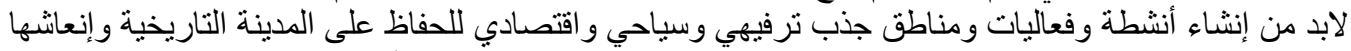

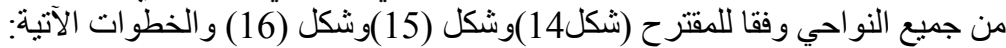

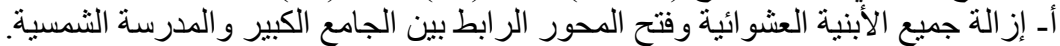

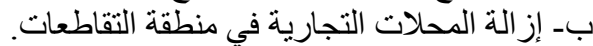

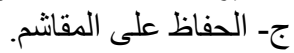
دـ- إنشاء نفق تحفت المحور المقاء و المنطقة الحضرية لغرض فصل حركة الآليات عن حركة الأشخاص. هـ إنشاء ساحة حضرية على امتداد المحور. و - إنشاء مركز للمدينة تتوفر فيه جميع المناء المقومات التجارية و الإدارية و الاستثمارية. 


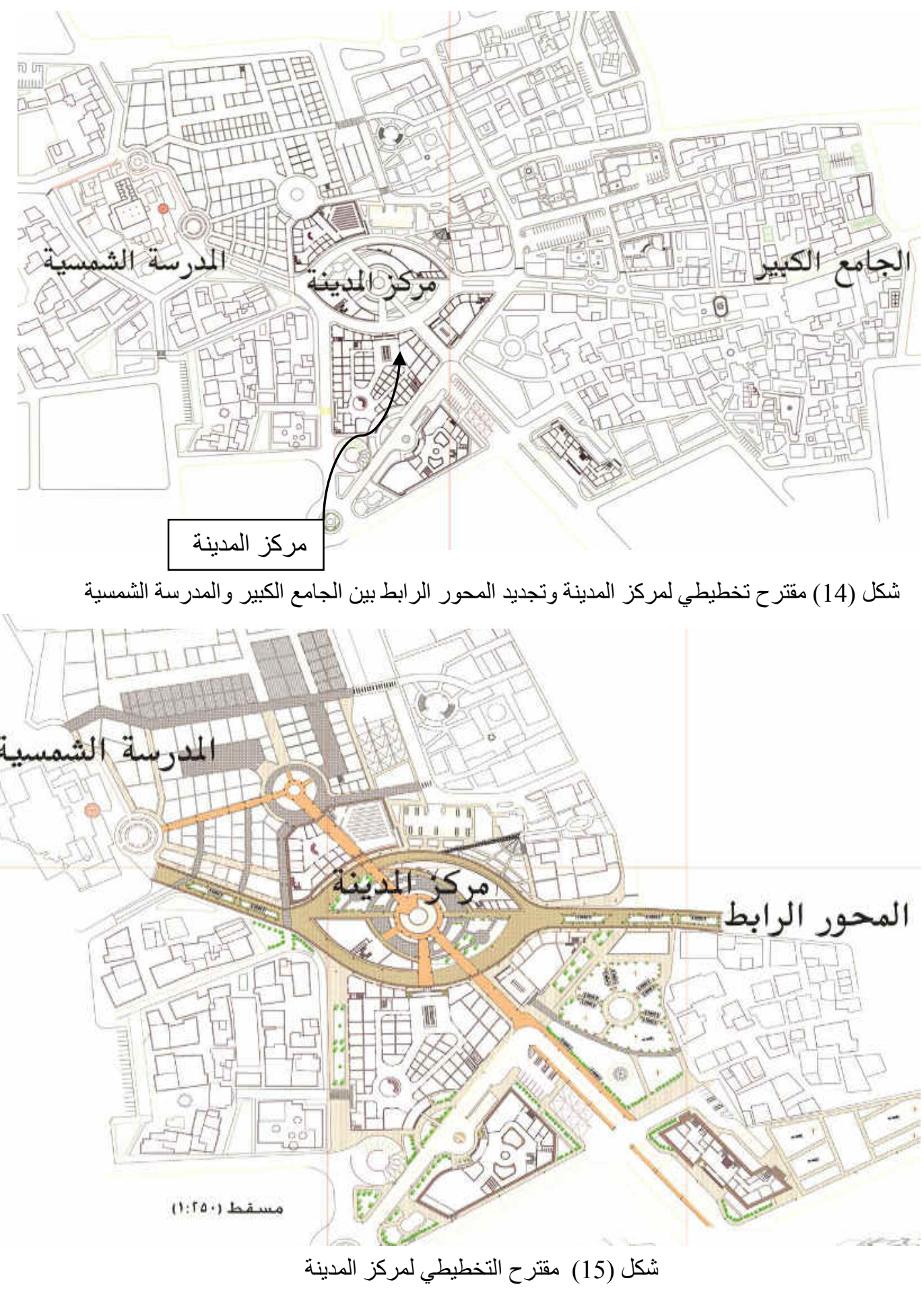




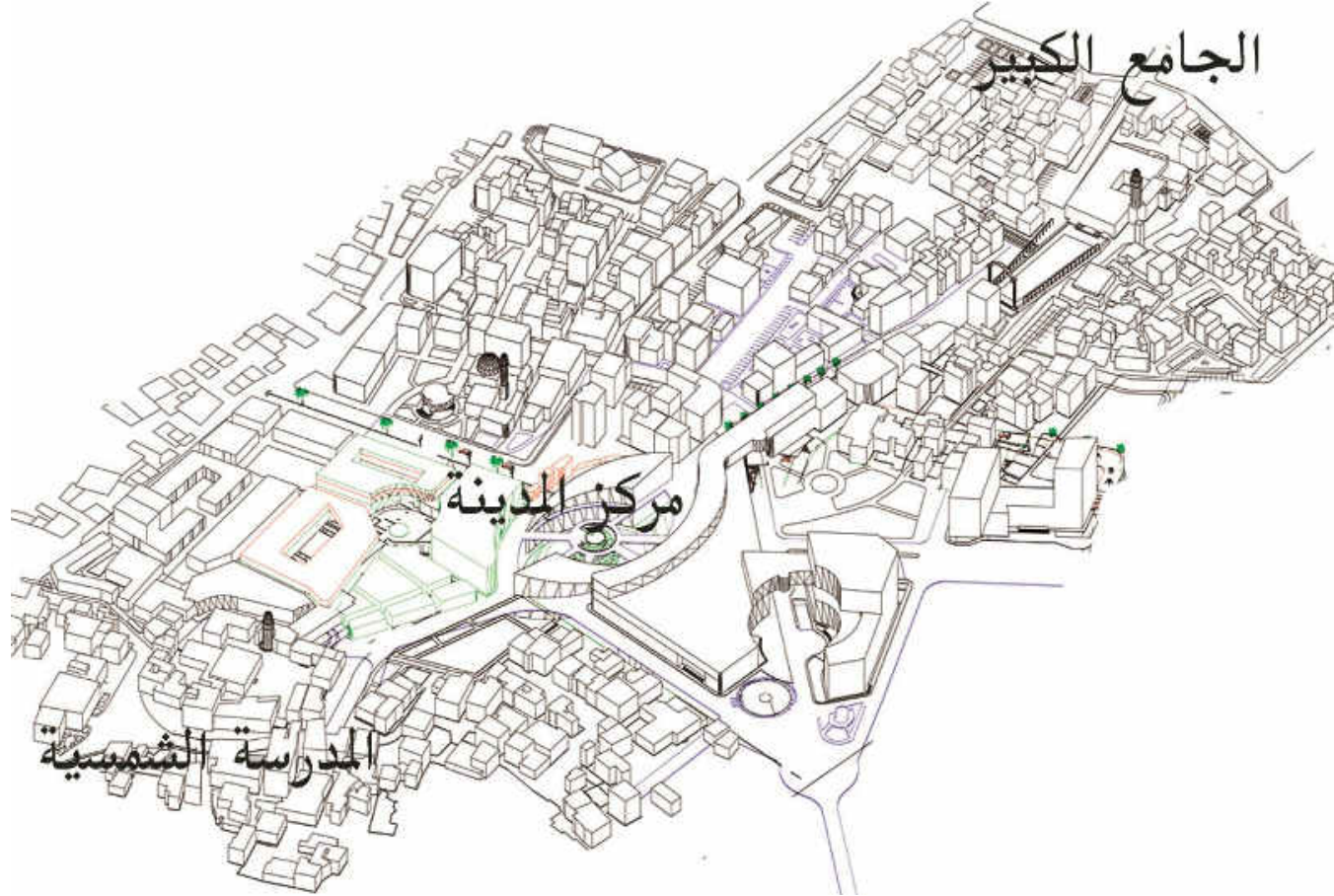

شكل (16) منظور للمقترح التخطيطي لمركز المدينة والمحور الرابط بين الجامع الكبير و المدرسة الثمسية

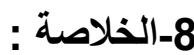

تعتبر مدينة ذمار التاريخية الثقافية ذات حجم صغير مساحيا وسكانيا, لكنها أخذت شهرتها التاريخية و العلمية

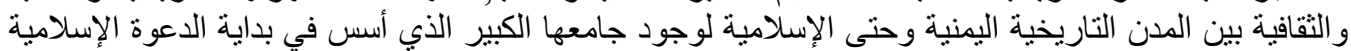

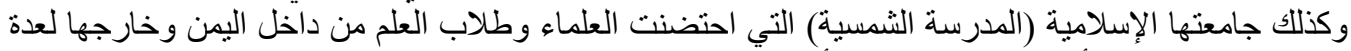

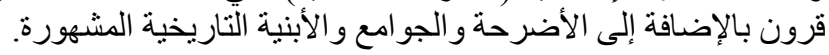

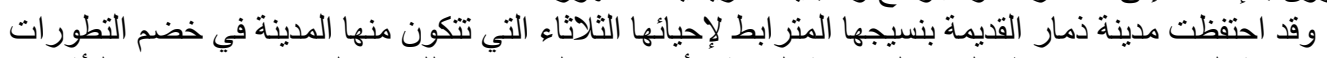

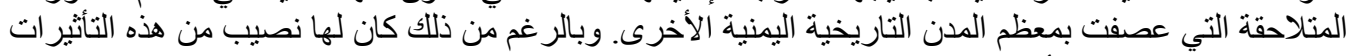

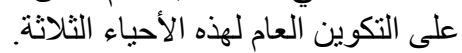

وومنذ بداية الثمانينات برزات الاحياء بوضونة أهمية الحفاظ على المدن التاريخية اليمنية وأطلقت الحملات المحلية

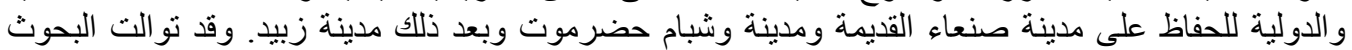

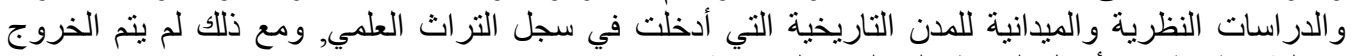

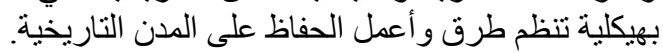

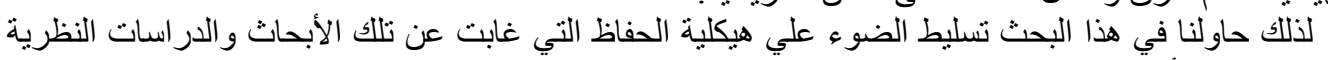
و التطبيقية في أعمال الحفاظ على البـ المدن التاريخية اليمنية. وتتسم مدينة ذمار بخاصية المدينة التقافية الحرفية التجارية الذية كانية كان يغلب على الإنى نسيجها العمر اني الساحات

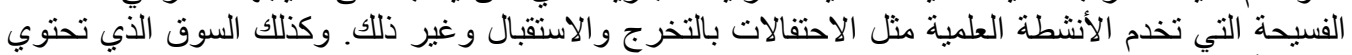

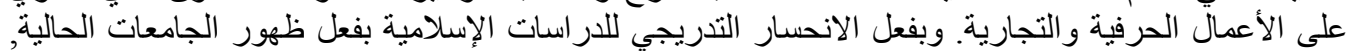

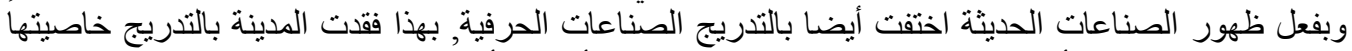

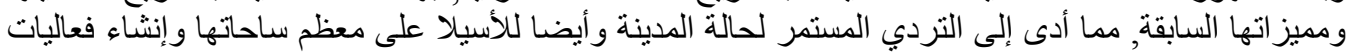

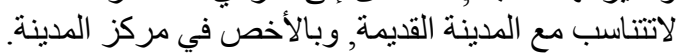

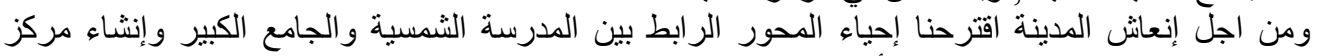
للمدينة بدلا عن المنطقة العشو ائية, من أجل إنعاش المدينة ثقافيا وسياسيا وتجاريا و إعادة الحياة للمدينة القديمة. 


\section{: النتائج}

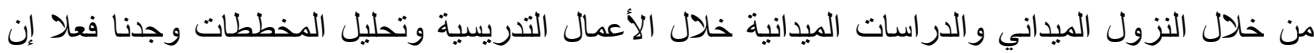

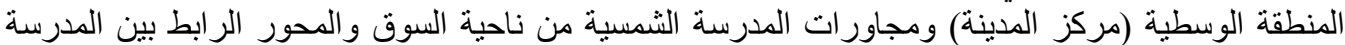

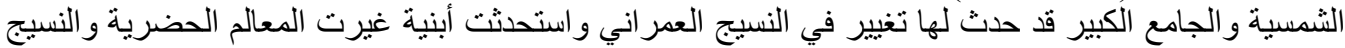
العمراني وبنفس الوقت أدى ذلك إلى اختفاء المحور الرابط بين الجامع الكبير والمدرسة الثمسية. وعلى فئى هذا

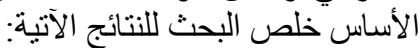

أـ تم إعادة تخطيط مركز المدينة والأجز اء المية المرتبطة بالمحور الر ابط بين الجامع الكبير و المدرسة الثمسية.

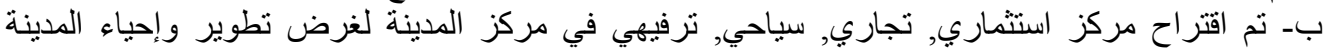

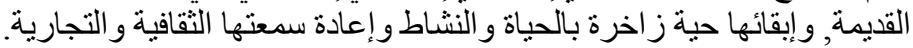

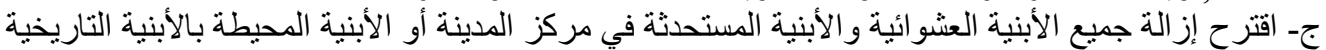

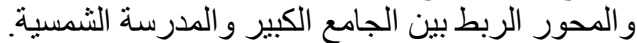

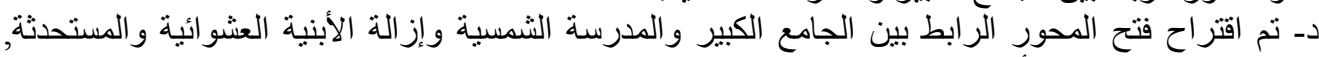
بالإضافة إلى تجديد الأبنية على هذا المحور.

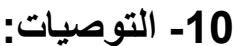

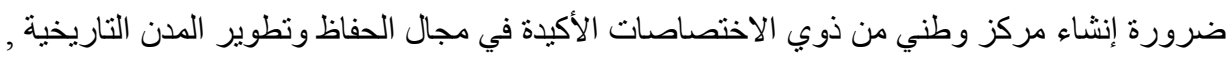
يكون قادر على وضع السياسات و الضو ابط , لتحديد مجال الحفاظ في كل مدينة و اتجاه تطور ها , و وتحديد مناطق التجديد و الإنعاثى ضرورة تحديد الأبنية والاستحداثيات الإنات التي أدت إلى تشويه وتعطيل النسيج الحضري (وإيقافها إلى حين توفر الإمكانيات في حالة عدم القدرة علي تنفيذ ذلك في الوقت الحالي) في المدن التاريخية.

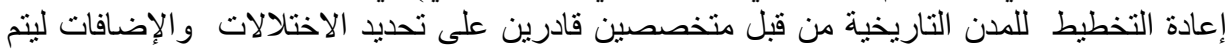

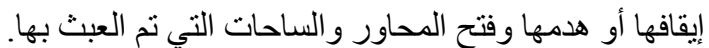
ضرورة تحديد مناطق الإنعاش و الاستثمار و السياحة و الترفية التهاح التي تخدم الحفاظ و التطوير.

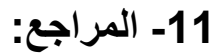

1- مجمو عة مر اجع تخطيط المدن , تصميم المدن المجلد الخامس , الكتاب الأول و الثاني , منشور ات دار

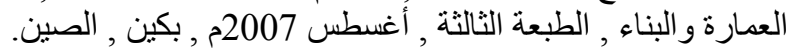

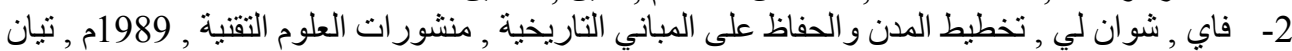

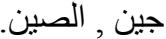
3- مجلة " الفضناء المثالي " منشورات جامعة التو نجي , العدد 29 نوفمبر , 2007م , شنغهابي , الصين.

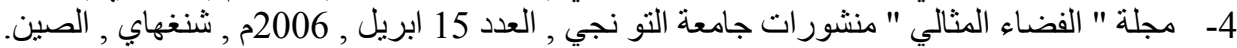

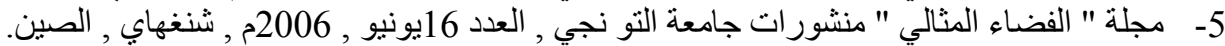
6- مجلة " جامعة الجنوب الشرقي " , العدد 16يونيو , 2000م , نانجين , الصين.

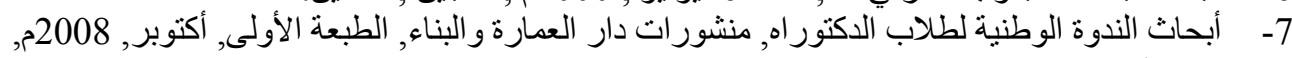
بكين, الصين.

8- العبسي , د/ عبد ثابت , تخطيط مدينة زبيد التاريخية بين الحفاظ و التنمية , مجلة كلية الهندسة جامعة صنعاء, المجلد الأول العدد رقم(2)- ديسمبر 2004.

\section{2- هوامش الكتب والرسائل العلمية والأبحاث:}

[1] الجر افي, عبد الله ناصر, مدينة ذمار, در اسة في جغرافية المدن, ماجستير غير منشورة , جامعة ذمار, 2004.

[2] الحجري اليماني, محمد بن أحمد, تحقيق وتصحيح لأكوع, إسماعيل بن علي, بلدان اليمن وقبائلها, المجلد الأول, صري 143, مكتبة الإرشاد, صنعاء, 2004/1/19م. 
[3] المقطري, نجيب علي عبد الله, الحفاظ و إعادة تأهيل الأحياء السكنية في المدن التاريخية:إعادة تأهيل وتنسيق

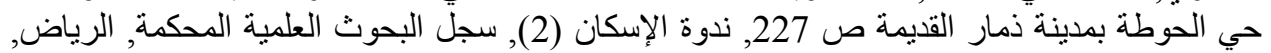
2004م.

[4] الحجري اليماني - مرجع سابق. [5] الدر استة الميدانية بالاعثماد على الخر ائط وتتبع الحقب التاريخية التي مرت بها المدينة والحالة الحالية, ومن

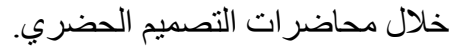

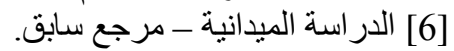

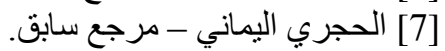

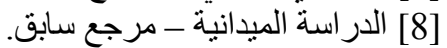
[9] [الدر اسة الميدانية - مرجع سابق.

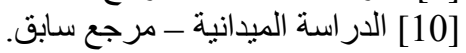
[11] [11 الدر اسة الميدانية - مرجع سابق.

\section{3- هوامش الأسماء:}

(1*) الساحات عبارة عن فضاء انفتاحي أمام مداخل الأبنية أو بين مسافات متفاوتة من الثوارع الرئيسية أو الفر عية.

(2*) المقاشم عبارة عن فضاء واسع تحيط به الأبنية السكنية, ومن وظائفه تموين المدينة بالخضروات (3*) المتنفسات عبارة عن فضاء بين الأبنية والأبنية الأخرى, لكي يعطي إطلالة وتهوية وإبراز أهمية المبنى.

\section{4- الأشكال:}

1 - (شكل 2 ) بالاعتماد على:

الجر افي, عبد الله ناصر, مدينة ذمارئ دادر دراسة في جغر افية المدن ص 57, ماجستير غير منشورة - جامعة

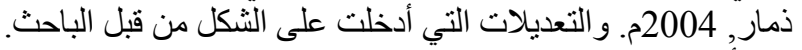

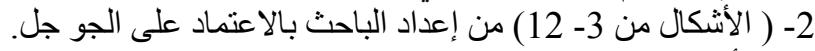
3- (الأشكال من 13- 16 16) بالاعتماد على محاضر ات التصاد التصميم الحضري. 